\title{
Combinatorial Benders' Cuts for Mixed-Integer Linear Programming
}

\author{
Gianni Codato and Matteo Fischetti \\ DEI, University of Padova, Italy \\ e-mail: matteo.fischetti@unipd.it
}

May 24, 2004; Revised, February 16, 2005

\begin{abstract}
Mixed-Integer Programs (MIP's) involving logical implications modelled through big-M coefficients, are notoriously among the hardest to solve. In this paper we propose and analyze computationally an automatic problem reformulation of quite general applicability, aimed at removing the model dependency on the big-M coefficients. Our solution scheme defines a master Integer Linear Problem (ILP) with no continuous variables, which contains combinatorial information on the feasible integer variable combinations that can be "distilled" from the original MIP model. The master solutions are sent to a slave Linear Program (LP), which validates them and possibly returns combinatorial inequalities to be added to the current master ILP. The inequalities are associated to minimal (or irreducible) infeasible subsystems of a certain linear system, and can be separated efficiently in case the master solution is integer. The overall solution mechanism resembles closely the Benders' one, but the cuts we produce are purely combinatorial and do not depend on the big-M values used in the MIP formulation. This produces an LP relaxation of the master problem which can be considerably tighter than the one associated with original MIP formulation. Computational results on two specific classes of hard-to-solve MIP's indicate the new method produces a reformulation which can be solved some orders of magnitude faster than the original MIP model.
\end{abstract}

Key words: Mixed-Integer Programs, Benders' Decomposition, Branch and Cut, Computational Analysis. 


\section{Introduction}

We first introduce the basic idea underlying combinatorial Benders' cutsmore elaborated versions will be discussed in the sequel.

Suppose one has a basic 0-1 ILP of the form

$$
\min \left\{c^{T} x: F x \leq g, x \in\{0,1\}^{n}\right\}
$$

amended by a set of "conditional" linear constraints involving additional continuous variables $y$, of the form

$$
x_{j(i)}=1 \Rightarrow a_{i}^{T} y \geq b_{i}, \text { for all } i \in I
$$

plus a (possibly empty) set of "unconditional" constraints on the continuous variables $y$, namely

$$
D y \geq e
$$

Note that the continuous variables $y$ do not appear in the objective function - they are only introduced to force some feasibility properties of the $x$ 's.

A familiar example of a problem of this type is the classical Asymmetric Travelling Salesman Problem with time windows. Here the binary variables $x_{i j}$ are the usual arc variables, and the continuous variables $y_{i}$ give the arrival time at city $i$. Implications (2) are of the form

$$
x_{i j}=1 \Rightarrow y_{j} \geq y_{i}+\text { travel_time }(i, j)
$$

whereas (3) bound the arrival time at each city $i$

$$
\text { early_arrival_time }(i) \leq y_{i} \leq \text { late_arrival_time }(i) \text {. }
$$

Another example is the map labelling problem [29], where the binary variables are associated to the relative position of two labels to be placed on a map, the continuous variables give their placement coordinates, and the conditional constraints impose non-overlapping conditions of the type "if label $i$ is placed on the right of label $j$, then the placement coordinates of $i$ and $j$ must obey a certain linear inequality giving a suitable separation condition".

The usual way implications (2) are modelled within the MIP framework is to use the (in)famous big-M method, where large positive coefficients $M_{i}$ are introduced to activate/deactivate the conditional constraints as in:

$$
a_{i}^{T} y \geq b_{i}-M_{i}\left(1-x_{j(i)}\right) \text { for all } i \in I
$$

This yields a (often large) mixed-integer model involving both $x$ and $y$ variables - whereas, in principle, $y$ variables are just artificial variables. 
Even more importantly, due to the presence of the big-M coefficients, the LP relaxation of the MIP model is typically very poor. As a matter of fact, the $x$ solutions of the LP relaxation are only marginally affected by the addition of the $y$ variables and of the associated constraints. In a sense, the MIP solver is "carrying on its shoulders" the burden of all additional constraints and variables in (2)-(3) at all branch-decision nodes, while they becomes relevant only when the corresponding $x_{j(i)}$ attains value 1 (typically, because of branching).

Of course, we can get rid of the $y$ variables by using Benders' decomposition [5], but the resulting cuts are weak and still depend on the big-M values. As a matter of fact, the classical Benders' approach can be viewed as a tool to speed-up the solution of the LP relaxation, but not to improve its quality.

The idea behind "combinatorial" Benders' cuts is to work on the space of the $x$-variables only, as in the classical Benders's approach. However, we model the additional constraints (2)-(3) through the following Combinatorial Benders' (CB) cuts:

$$
\sum_{i \in C} x_{j(i)} \leq|C|-1
$$

where $C \subseteq I$ induces a Minimal (or Irreducible) Infeasible Subsystem (MIS, or IIS, for short) of (2)-(3), i.e., any inclusion-minimal set of row-indices of system (2) such that the linear subsystem

$$
S L A V E(C):=\left\{\begin{array}{c}
a_{i}^{T} y \geq b_{i}, \text { for all } i \in C \\
D y \geq e
\end{array}\right.
$$

has no feasible (continuous) solution $y$.

A CB cut is violated by a given $x^{*} \in[0,1]^{n}$ if and only if $\sum_{i \in C}\left(1-x_{j(i)}^{*}\right)<$ 1. Hence the corresponding separation problem essentially consists of the following steps: (i) weigh each conditional constraint $a_{i}^{T} y \leq b_{i}$ in (2) by $1-x_{j(i)}^{*}$; (ii) weigh each unconditional constraint in (3) by 0 ; and (iii) look for a minimum-weight MIS of the resulting weighted system - a NP-hard problem $[1,17]$.

A simple polynomial-time heuristic for CB-cut separation is as follows. Given the (fractional or integer) point $x^{*}$ to be separated, start with $C:=$ $\left\{i \in I: x_{j(i)}^{*}=1\right\}$, verify the infeasibility of the corresponding linear subsystem $S L A V E(C)$ by classical LP tools, and then make $C$ inclusion-minimal in a greedy way. Though extremely simple, this efficient separation turns out to be exact when $x^{*}$ is integer.

The discussion above suggests the following exact Branch \& Cut solution scheme. We work explicitly with the integer variables $x$ only. At each branching node, the LP relaxation of a master problem (namely, problem (1) amended by the CB cuts generated so far) is solved, and the heuristic 
CB separation is called so as to generate new violated CB cuts (and to assert the feasibility of $x^{*}$, if integer).

The new approach automatically produces a sequence of CB cuts, which try to express in a purely-combinatorial way the feasibility requirement in the $x$ space - the CB cut generator acting as an automatic device to distill more and more combinatorial information from the input model. As a consequence of the interaction among the generated CB cuts, other classes of combinatorial cuts are likely to be violated, hence allowing other cut separators to obtain a further improvement. We found that the $\left\{0, \frac{1}{2}\right\}$-cuts addressed in $[9,2]$ fit particularly well in this framework, and contribute substantially to the overall efficacy of the approach.

It is worth noting that, using the new technique, the role of the big-M terms in the MIP model vanishes-only implications (2) are relevant, no matter the way they are modelled. Actually, the approach suggests an extension of the MIP modelling language where logical implications of the type (2) can be stated explicitly in the model, as in Hooker and Osorio [21].

In this paper we aim at investigating whether the above method can be useful to approach certain types of MIP's which are notoriously very hard to solve. As shown in the computational section, this is indeed the case: even in its simpler implementation, on some classes of instances the new approach allows for a speed-up of some orders of magnitude with respect to ILOG-Cplex 8.1, one of the best MIP solvers on the market.

Our technique is based on Hooker's idea of deriving Benders' cuts from minimal sets of inconsistencies, as proposed in [19]. In this respect, our main contributions have been (a) to present a separation heuristic that finds a minimal Benders' cuts for the special case of conditional constraints with linear implications, and (b) to test these cuts computationally on some hard MIP problems. This is an important special case because conditional constraints of this form are a very useful modeling device.

The paper is organized as follows. In Section 2 we present the new approach in a more general context, whereas previous literature on the subject is reviewed in Section 3. As already stated, our CB cut separator requires a fast determination of MIS's; this important topic is addressed in Section 4, where an approach particularly suited to our application is described. Computational results are presented in Section 5, with the aim of verifying whether a simple implementation of the new method can already produce improved performance with respect to the application of a sophisticated MIP solver such as ILOG-Cplex 8.1 (at least, on some problem classes which fit particularly well in our scheme). Finally, some conclusions are drawn in Section 6.

The present paper is based on the master thesis of the first author [12], which was awarded the 2003 Camerini-Carraresi prize by the Italian Operation Research association (AIRO). Moreover, the paper was presented at the IPCO X meeting held in New York, June 2004. 


\section{Combinatorial Benders' cuts}

Let $P$ be a MIP problem with the following structure:

$$
\begin{aligned}
P: \quad z^{*}:=\min \quad & c^{T} x+d^{T} y \\
\text { s.t. } \quad & F x \leq g \\
& M x+A y \geq b \\
& D y \geq e \\
& x_{j} \in\{0,1\} \quad \text { for } j \in B \\
& x_{j} \text { integer } \quad \text { for } j \in G
\end{aligned}
$$

where $x$ is a vector of integer variables, $y$ is a vector of continuous variables, $G$ and $B$ are the (possibly empty) index sets of the general-integer and binary variables, respectively, and $M$ is a matrix with exactly one nonzero element for every row $i$, namely the one indexed by column $j(i) \in B$. In other words, we assume the linking between the integer variables $x$ and the continuous variables $y$ is only due to a set of constraints of the type

$$
m_{i, j(i)} x_{j(i)}+a_{i}^{T} y \geq b_{i} \text { for all } i \in I
$$

where variables $x_{j(i)}$ are binary for all $i \in I$.

We consider the case $d=0$ first, i.e., we assume the MIP objective function does not depend on the continuous variables, and leave case $d \neq 0$ for a later analysis. In this situation, we can split problem $P$ into two sub-problems:

- MASTER:

$$
\begin{array}{rc}
z^{*}=\min & c^{T} x \\
\text { s.t. } & F x \leq g \\
& x_{j} \in\{0,1\} \text { for } j \in B \\
& x_{j} \text { integer } \text { for } j \in G
\end{array}
$$

- $S L A V E(\tilde{x})$, a linear system parametrized by $\tilde{x}$ :

$$
\begin{gathered}
A y \geq b-M \tilde{x} \\
D y \geq e
\end{gathered}
$$

Let us solve the master problem at integrality. If this problem turns out to be infeasible, then $P$ also is. Otherwise, let $x^{*}$ be an optimal solution (we exclude the unbounded case here, under the mild assumption that, e.g., the general-integer variables are bounded). 
If the linear system $S L A V E\left(x^{*}\right)$ has a solution, say $y^{*}$, then clearly $\left(x^{*}, y^{*}\right)$ is an optimal solution of $P$. If the slave is infeasible, instead, $x^{*}$ itself is infeasible for problem $P$. We therefore look for a MIS of $S L A V E\left(x^{*}\right)$, involving the rows of $A$ indexed by $C$ (say), and observe that at least one binary variable $x_{j(i)}$ has to be changed in order to break the infeasibility. This condition can be translated by the following linear inequality in the $x$ space, that we call Combinatorial Benders' (CB) cut:

$$
\sum_{i \in C: x_{j(i)}^{*}=0} x_{j}+\sum_{i \in C: x_{j(i)}^{*}=1}\left(1-x_{j}\right) \geq 1 .
$$

One or more $\mathrm{CB}$ cuts of this type are generated in correspondence of a given infeasible $x^{*}$, and added to the master problem. Iterating the procedure produces an exact solution method in the spirit of Benders' decomposition.

Of course, it is advisable to exploit the CB cuts within a modern Branch \& Cut scheme which hopefully produces violated cuts at each node of the branching tree - and not just in correspondence of an integer optimal solution of the master. Note that the correctness of the Branch \& Cut method only requires the generation of a violated $\mathrm{CB}$ cut (if any) before each updating of the incumbent solution of the master, i.e., any heuristic CB separator that guarantees to be exact for an integer $x^{*}$ already suffices to get a valid solution method.

We now address the case $c=0$ and $d \neq 0$, arising when the objective function only depends on the continuous variables $y$. In this situation, we cannot accommodate the objective function into the master problem. Instead, we can add the bound inequality $d^{T} y \leq U B-\epsilon$ to the slave system, where $U B$ is the value of the incumbent solution, and $\epsilon$ is a sufficiently small positive value. In this way, the $\mathrm{CB}$ cuts will translate both the feasibility and the optimality requirements. More specifically, at the iterations where $S L A V E\left(x^{*}\right)$ (amended by the bound inequality) is infeasible, we can generate one or more violated $\mathrm{CB}$ cuts, as required. At the iterations where this system is feasible, instead, we can find an optimal solution $y^{*}$ of the LP problem $\min \left\{d^{T} y: A y \geq b-M x^{*}, D y \geq e\right\}$ and update the best incumbent solution by $\left(x^{*}, y^{*}\right)$. The overall method will stop when the current master (that now looks for a feasible $x$ improving the incumbent) becomes infeasible. Finally, we observe that the case $c \neq 0$ and $d \neq 0$ cannot be dealt with effectively by our method. A possible approach is to make an external binary search of the (unknown) value of $d^{T} y^{*}$ in an optimal solution $\left(x^{*}, y^{*}\right)$ of $P$, and exploit this information by introducing bound constraints of the type $d^{T} y=d^{T} y^{*}$ into the slave. However, this naive approach would imply the solution of a (possibly long) series of MIP's, hence its practical effectiveness should be investigated computationally - this topic is left to future research.

At first glance, the required assumptions - in particular, (14) - restrict the range of applicability of our method considerably. However, there are 
several important (and difficult) MIP's which fit naturally in our scheme. Some of them will be addressed in the computational section. In addition, there is a simple reformulation method that can extend its applicability significantly. The idea is to introduce a (continuous) copy $x_{j}^{c}$ of each binary variable $x_{j}, j \in B$, and to link the two copies through the constraints:

$$
x_{j}=x_{j}^{c} \quad \text { for } j \in B
$$

By construction, the above constraints can play the role of (14), thus linking the master problem to the slave.

In particular, one can always reformulate a generic MIP with no generalinteger variables (i.e., involving only binary and continuous variables) as follows. Introduce the continuous copy $x^{c}$ of the entire vector $x$. The initial master takes the binary variable vector $x$, plus the constraints $F x \leq g$ along with the obvious constraints $x \in\{0,1\}^{n}$; the master objective function is zero (or $c^{T} x$ if $d=0$ ). The slave keeps the continuous variable vectors $x^{c}$ and $y$, the constraints $M x^{c}+A y \geq b, D y \geq e$ along with the linking equations $x=x^{c}$, plus the objective function bound $c^{T} x^{c}+d^{T} y \leq U B-\epsilon$, where $U B$ is the value of the incumbent solution.

Actually, one can even decide to remove $F x \leq g$ from the master, and to introduce $F x^{c} \leq g$ into the slave (the master objective function being zero). With this choice, at each iteration the master only contains the distilled combinatorial information (notably, CB cuts) that exclude certain configurations of the binary variables - because they are infeasible or cannot lead to an improved solution. The master then iteratively detects new binary solutions $x^{*}$ which fulfill the master constraints generated so far, and invokes the slave for validation. The slave verifies the feasibility of the proposed $x^{*}$ (with respect to the LP relaxation of the original MIP, amended by the upper bound constraint), possibly updates the incumbent, and then returns one or more $\mathrm{CB}$ cuts related to some forbidden minimal configurations of the binary variables.

\section{Related work}

CB cuts have their roots in the seminal work of Hooker [19] on logic-based methods for optimization, where the problem under investigation has the very general form

$$
\begin{array}{rc}
\operatorname{minf}(x) & \\
\text { s.t. } & p_{i}(x), i \in I_{1} \\
& g_{i}(y), i \in I_{2} \\
& q_{i}(x) \Rightarrow h_{i}(y), \quad i \in I_{3}
\end{array}
$$

If $\mathrm{C}$ is a minimal conflict set, in the sense that $\mathrm{C}$ is an inclusion-minimal subset of $I_{3}$ such that $\left\{h_{i}(y): i \in C\right\} \cap\left\{g_{i}(y): i \in I_{2}\right\}$ is infeasible, then 
one can write the Benders' cut condition

$$
\bigvee_{i \in C} \neg q_{i}(x)
$$

In this context, our approach deals with the special case where conditions $p_{i}(x)$ are linear inequalities in the integer variables $x_{j}$, the $g_{i}(y)$ and $h_{i}(y)$ are linear inequalities, and the $q_{i}(x)$ have the form $x_{j(i)}=1$. Indeed, in this case condition $(27)$ can be rephrased as the CB cut $\sum_{i \in C}\left(1-x_{j(i)}\right) \geq 1$.

Apparently, the first practical use of this kind of Benders' cuts was to solve circuit verification problems [23]. In this application the subproblem constraints $h_{i}(y)$ are again linear inequalities (actually, systems of linear inequalities), but they have a special structure that permits the rapid identification of minimal conflict sets. Hooker studied logic-based Benders' cuts in [19] and proposed applying them to multiple machine scheduling, in which constraints $h_{i}(y)$ are scheduling constraints rather than linear inequalities. Jain and Grossmann [26] implemented this idea and obtained very good results - even without insisting on finding minimal conflict sets. Similar results were obtained for a broader class of problems in [20], but these results required a more sophisticated form of Benders' cut than (27). Cambazard et al. [8] returned to the idea of min-conflict Benders' cuts to solve real-time scheduling problems on multiple machines. They relied on a min-conflict algorithm of Junker [27], which improves on an earlier algorithm of De Siqueira and Puget [13].

Kim and Hooker [28] considered fixed-charge network flow problems, and proposed a scheme that is very similar to the approach proposed in this paper. The main difference is that Kim and Hooker find minimal infeasible configurations of the binary variables (the so-called nogoods) specifically in a min-cost network flow relaxation, while this paper finds them in a more general context. The same comment applies to the map labelling problem studied by Mützel and Klau [29]; see Section 5 for details.

Rather than alternating between solving a master problem and subproblem, as in the classical Benders' method, our approach solves a single master problem and generate Benders' cuts on the fly. This approach was proposed in [19] and later named "branch and check" by Thorsteinsson [32], who successfully applied it to the Jain and Grossmann problems. Meanwhile, Hooker and Ottosson [22] used logic Benders' cuts to solve SAT problems and $0-1$ programming problems.

Our solution method also has interesting connections with Chvátal's resolution search. Roughly speaking, resolution search can be viewed as an attempt to get rid of the rigid tree paradigm used within enumeration schemes. Convergence of generic enumerative methods for combinatorial optimization problems requires to record information about the subsets of the solution space that have been already explored - so as to avoid considering twice a same subset, and to abort the search when no unexplored subset exists. For 
each subset, an oracle (borrowing Chvátal terminology) is invoked to answer the basic question: Is there any hope that this subset contains a solution improving the incumbent? If (possibly after having updated the incumbent) the answer is "no", the subset is discarded. If the answer is "yes", the set is subdivided further. For MIP problems, the subsets are typically defined by a branching strategy, i.e., by fixing the value of certain subsets of the integer variables. Resolution search can be viewed as a way to control the solution process. Here, the standard branching tree is replaced by a pool of logical conditions that summarize the previous computation. At each iterations, a partial assignment of the binary variables is found, which fulfills the logical conditions in the current pool. If no such assignment exists, then the enumeration is over and the incumbent is a provable optimal solution. Otherwise, the oracle is invoked in two distinct greedy phases. In the waxing phase, the current partial assignment is iteratively extended up to a point where the oracle returns a "no" answer (with the incumbent possibly updated). In the subsequent waning phase, the current partial assignment is iteratively reduced as long as the oracle answer remains "no". At the end of the waning phase, the current partial assignment corresponds to an obstacle, i.e., to a minimal set of variables that have to be changed in order to get a "yes" answer from the oracle. The logical condition "change the value at least one of the variables in the obstacle" is then added to the pool of logical conditions, so as to avoid to face the same obstacle in the later exploration, and the process is iterated. The analogy with our approach is now evident: our master problem plays the role of a 0-1 ILP model for the logical conditions in the pool, whereas the slave is our implementation of the oracle function to detect one or more obstacles efficiently.

There is however an important difference between our method and resolution search. Indeed, in resolution search the nogoods are structured so that one can find a feasible solution for the accumulated nogoods in polynomial time. In Chvátal method, in particular, the nogoods have a proper pathlike structure; other variations on this theme include dynamic backtracking, dependency-directed backtracking, partial-order dynamic backtracking, and generalized partial-order dynamic backtracking [7, 14, 15, 16, 19]. This nice feature is not preserved in our method, where the master problem is a (somehow general) NP-hard ILP.

$\mathrm{CB}$ cuts can be generated within a standard Branch \& Cut solution framework not based explicitly on the master/slave decomposition we propose. For the sake of notation, let us consider a pure 0-1 ILP of the form $\min \left\{c^{T} x: A x \geq b, x \in\{0,1\}^{n}\right\}$. Take a generic branching node $N O D E_{h}$ which is fathomed by the classical lower bound criterion, possibly after the updating of the incumbent solution. The node corresponds (say) to the fixing $x_{j}=x_{j}^{*}$ for some $j \in J_{h}$, where $x_{j}^{*} \in\{0,1\}$ is the known branching value for variable $x_{j}$ at $N O D E_{h}$. The fathoming condition then implies that the slave linear system made by the "linking equations" $x_{j}=x_{j}^{*}$ for $j \in J_{h}$ and 
by the inequalities $A x \geq b, x \in[0,1]^{n}, c^{T} x \leq U B-\epsilon$, is infeasible. Finding a MIS of this system then yields an inclusion-minimal $C \subseteq J_{h}$ whose associate $\mathrm{CB}$ cut (21) can be added to the current formulation. (Of course, in this context the new cut is only useful if $C$ is a proper subset of $J_{h}$, so as to exclude a solution subset strictly larger than the one associated with the current node $N O D E_{h}$.) Our approach can therefore be viewed as a simple (yet hopefully effective) method for "branching resequencing" in the spirit of the Dynamic Branch and Bound of Glover and Tangedhal [18].

\section{Fast MIS search}

In this section we describe an efficient algorithm to find a MIS of an infeasible linear system, that fits particularly well within our solution approach. The method is in the spirit of the one discussed by Parker and Ryan in [30]

MIS search can be formulated as follows: given an infeasible system of inequalities, say $\tilde{A} y \geq \tilde{b}$, find an inclusion-minimal set of its rows yielding an infeasible system.

We therefore construct the LP

$$
\begin{aligned}
\min & 0^{T} y \\
\text { s.t. } & \tilde{A} y \geq \tilde{b}
\end{aligned}
$$

and its dual

$$
\begin{array}{cc}
\max & u^{T} \tilde{b} \\
\text { s.t. } & u^{T} \tilde{A}=0^{T} \\
& u \geq 0
\end{array}
$$

It is known that if the primal problem is infeasible, then the corresponding dual can be either unbounded or infeasible. Now, dual infeasibility is excluded by the fact that $u=0$ is always dual feasible, hence primal infeasibility corresponds to dual unboundedness, i.e., to the existence of a dual solution $u^{*}$ such that $u^{* T} \tilde{b}>0$ (hence $k u^{*}$ for a sufficiently large $k>0$ is a feasible dual solution with arbitrarily large objective value). Therefore we can replace the dual objective function by the following constraint:

$$
u^{T} \tilde{b}=1
$$

This modified dual problem is meant at finding a linear combination of the rows of $\tilde{A} y \geq \tilde{b}$ leading to a valid inequality $u^{* T} \tilde{A} y \geq u^{* T} b$ with all-zero left-hand side coefficients and strictly positive right-hand side, thus proving the infeasibility of $\tilde{A} y \geq \tilde{b}$ - the existence of $u^{*}$ is guaranteed, for infeasible systems, by the Farkas' lemma.

It is known [17] that each vertex of the dual polyhedron defined by (31)(33) has a support defining an MIS (whereas minimality is not guaranteed for 
an arbitrary point $u$ of the same polyhedron). In our applications, we look for a solution $u^{*}$ having a small support in the set of the linking constraints (19), whereas we do not actually care on the minimality with respect to (20). This suggests the use of the heuristic dual objective function $\sum_{i} w_{i} u_{i}$, where weights $w_{i}$ 's are used to drive the LP solver to select a solution $u^{*}$ with the desired characteristics. Moreover, by iteratively setting to zero some of the $u$ variables we can easily detect alternative MIS's, which is very important in order to generate several violated CB cuts at each call of the separation heuristic.

According to our computational experience, the described algorithm is very effective and outperforms (for our applications) an analogous MIS algorithm based on the ILOG-Cplex function IloCplex: : getIIS().

\section{Computational Results}

To evaluate its effectiveness, we implemented our method in $\mathrm{C}++$ and embedded it within the ILOG-Cplex Concert Technology 1.2 framework, based on ILOG-Cplex $8.1[24,25]$.

In our implementation, we read an input MIP in the form (8)-(13), and verify that (a) the linking constraints are of the form (14) with binary $x_{j(i)}$, and (b) the objective function only depends on the integer variables. We then construct (automatically) the master/slave decomposition, and invoke the ILOG-Cplex solver on the master. During the ILOG-Cplex Branch \& Cut execution, we call our separation procedures for $\mathrm{CB}$ and $\left\{0, \frac{1}{2}\right\}$-cuts. In the $\mathrm{CB}$ separation, the integer components of the current master LP solutions $x^{*}$ are sent to the slave, in the attempt to generate one or more CB cuts through the MIS search described in Section 3. As to $\left\{0, \frac{1}{2}\right\}$-cuts, we use the separation code of Andreello, Caprara, and Fischetti [2]. This separation proved quite effective, and allowed for a reduction of up to $50 \%$ of the computing time of our method for some instances of the test-bed. (Observe that effective $\left\{0, \frac{1}{2}\right\}$-cuts cannot be derived from the original MIP formulation of the instances in our test-bed, due to the presence of the continuous variables.)

In order not to overload the LP formulation, we avoided calling our CB and $\left\{0, \frac{1}{2}\right\}$-cut separators at each node of the branching tree, but applied them (a) before each updating of the incumbent solution; (b) at each node with a tree depth not greater than 10; and (c) after each backtracking step (see [2] for a discussion on similar strategies).

Due to the heuristic nature of our separation procedures for $\mathrm{CB}$ and $\left\{0, \frac{1}{2}\right\}$-cuts, and since the number of generated cuts tends to increase steeply, all cuts are stored in a constraint pool, which is purged from time to time.

It should be observed that ILOG-Cplex 8.1 does not implement an internal cut pool: once a (globally valid) cut has been generated at run time, it 
is added statically to the LP formulation and never removed. An important exception (that we exploit in our code) arises for locally-valid cuts, which are automatically removed from the formulation the first time they are no longer guaranteed to be valid (because of a backtracking step); removed cuts are not saved.

The lack of a native pool within ILOG-Cplex 8.1 is not really an issue in itself, in that one can easily implement an external data structure to store the $\mathrm{CB}$ and $\left\{0, \frac{1}{2}\right\}$-cuts. A possible issue is however the impossibility of removing a (globally valid) cut from the current LP. This makes it impossible to purge the current LP by removing some of its constraints, with the risk it becomes soon too large. A possible work-around is to define the $\mathrm{CB}$ and $\left\{0, \frac{1}{2}\right\}$-cuts as local (as opposed to global) cuts, so they are automatically removed from time to time from the LP. Though this approach does not allow us to have a complete control on the cut removal mechanism (nor on the the size of the LP), it works reasonably well in practice - although it implies a certain memory overhead.

\subsection{The test-bed}

Realistically, one cannot expect our approach works well in all applications. Indeed, CB cuts can sometimes be of very weak polyhedral quality, hence they do not capture in an adequate way the complexity of the problem at hand. In particular, this is the case for the Asymmetric Travelling Salesman Problem with time windows (the problem we used as an introductory example), where CB cuts can only state the single infeasibility of certain paths and are dominated by much stronger classes of problem-specific inequalities, including the tournament inequalities studied in $[3,4]$. However, our method proved to have some merits in handling difficult MIP problems where CB cuts play a role in describing in a strong polyhedral way the underlying combinatorial structure. Examples of this kind of problems are described next.

Map Labelling. This problem consists in placing as many rectangular labels as possible (without overlap) in a given map. If placed, a label has a limited degree of movement around its associated "reference point" (a prespecified point of the map corresponding to, e.g., the city whose name is in the label). This problem has been formulated as a MIP model involving big-M coefficients by Mützel and Klau [29], who report very poor results when trying to solve the model directly. Much better results are in fact obtained by the same authors when using a different (purely combinatorial) 0-1 ILP model, where the binary variables are associated with arcs of a suitable digraph, and the non-overlapping conditions are translated by rank inequalities forbidding the choice of all the arcs in a circuit with certain properties. Actually, it was precisely the nice correspondence between the 
MIP model and its graph reformulation that motivated us to generalize the Mützel-Klau construction, thus originating the work presented in the present paper. As a matter of fact, applying our method to their MIP model produces automatically a slave problem with a network structure, hence it can be associated with a digraph whose circuits (under appropriate conditions) correspond to minimal infeasible subsystems - and hence to CB cuts.

Our test-bed contains 18 map labelling instances, of the so-called 4-slider (4S) and 4-position (4P) type, kindly provided by G.W. Klau.

As shown in the computational section, the results we obtained on map labelling problems are comparable with those reported in [29], even though our method is more general and does not exploit the specific network structure of the slave. This is an indication that the overhead introduced in our implementation (in particular, for computing MIS's via LP methods rather than using fast graph-search algorithms) is acceptable.

Two-Group Statistical Classification (discriminant analysis). This problem can be described briefly as follow; see, e.g., Rubin [31] for more details. We have a population whose members can be divided into two distinct classes - for example, people affected or not by a certain disease. We can measure a number of characteristics that are related to the partition abovee.g, biological parameters that show the presence or absence of the disease. Based on the available measures, we want to obtain a linear function which allows us to decide whether a new member belongs to the first or second class. More specifically, we are looking for a linear function that minimize the number of misclassified members in the known sample ${ }^{1}$. This problem can be modelled as a MIP problem with big-M coefficients: the unknowns are the coefficients of the linear function, and for every member there is a binary variable used to deactivate the inequality in case of misclassification. The purpose is to minimize the number of deactivated inequalities, so the objective function is just the sum of the binary variables. (In [31] a slightly different MIP model is used, involving also a real-valued variables in the objective; this model can easily be transformed into the one we use in the present study.)

The raw data from which we have generated our instances has been taken from a public archive maintained at UCI [33], and converted to the final MIP model with a slightly modified version of a program kindly provided us by P. A. Rubin. This resulted into 38 instances coming from different real situations (i.e., disease classification, interpretation of physical phenomena,

\footnotetext{
${ }^{1}$ Designing optimal linear classifiers consists in minimizing (resp., maximizing) the number of misclassified (resp., correctly classified) members. Given the infeasible inequality system imposing correct classification of all members, the problem amounts to deleting the minimum number of inequalities to make the system feasible, or equivalently to finding a maximum feasible subsystem (MaxFS); see, e.g., [10, 1].
} 
animal behavior, etc.).

\subsection{Results}

Our experiments have been performed on a PC AMD Athlon 2100+ with 1 GByte RAM, and GNU/Linux (kernel 2.4) Operating System.

All the instances of our test-bed have been processed twice: the first time by solving the original MIP model through the commercial ILOG-Cplex 8.1 solver (with default settings), and the second by using our implementation of the master/slave decomposition based on $\mathrm{CB}$ cuts and $\left\{0, \frac{1}{2}\right\}$-cuts (still based on the ILOG-Cplex 8.1 library). A time-limit of 3 CPU hours was imposed for each run. In addition, we set the IloCplex: :NodeFileInd parameter to 3: this forces Cplex to store the active-node data into the hard disk when physical memory is exhausted (due to the efficiency of this mechanism, we had no memory problems even when handling branching trees much larger than $1 \mathrm{~GB}$ )

In the tables below, label $C B C$ refers to our code (based con CB cuts), whereas label Cplex refers to the standard ILOG-Cplex 8.1 solver. We compared the two codes in terms of execution times and/or integrality gaps computed with respect to the best integer solution found, on the following three instance subclasses:

1. instances solved to proven optimality by both CBC and Cplex

2. instances solved to proven optimality by CBC but not by Cplex

3. instances not solved (to proven optimality) by CBC nor by Cplex

Notice that there was no instance in our test-bed that was solved by Cplex but not by CBC.

For the first subset, Table 1 reports the instance names, the optimal objective values (opt), the Cplex and CBC computing times, their ratios and the number of branching nodes enumerated by Cplex and by CBC for the exact solution of the instances. On these instances, CBC turns out to be up to 3 orders of magnitude faster that Cplex. According to the final row of the table, Cplex ran for almost 8.5 hours to solve all the instances of this subset, while $\mathrm{CBC}$ requires about 24 minutes, i.e., the latter code was 21 times faster in processing the whole subset. As to the number of nodes enumerated by the two approaches, we note that a direct comparison of these figures is not immediate in that the two methods actually work on different formulations - hence processing a single node may require different computing times. In particular, for $\mathrm{CBC}$ the large number of generated cuts tends to slow-down the node processing significantly.

Figure 1 reports the graph of the best bound and of the incumbent solution values for Cplex and $\mathrm{CBC}$ executions on a sample instance in this subset. 
The results for the second subset of instances are given in Table 2. As in Table 1, for CBC we report the optimization time and the optimal objective value, while for Cplex we give the value of the incumbent solution (best sol.) and of the best bound (best bound), their percentage gap (gap (\%), computed as $100 \mid$ best_sol - best_boud|/best_sol), as well as the MBytes of memory used (mem. MB). For each instance, we report two distinct rows for Cplex execution: the first one refers to the time instant when CBC finished its computation, and the second row gives the situation when Cplex is aborted (time limit expired). Finally, in the last row of the two sections of the table we report the total computing time and the average Cplex gaps, computed both when CBC finished its execution (same t.), and at the end of its own computation (end).

Figure 2 gives an illustration of the Cplex and CBC behavior on two

\begin{tabular}{|l|r|c|c|r|r|r|}
\hline Subset 1 & & \multicolumn{2}{|c|}{ Exec. Times } & \multicolumn{2}{|c|}{ Ratio } & \multicolumn{2}{|c|}{ Nodes } \\
\hline File name & opt & $\begin{array}{c}\text { Cplex } \\
\text { h: } \mathrm{m}: \mathrm{s}\end{array}$ & $\begin{array}{c}\text { CBC } \\
\text { h: } \mathrm{m}: \mathrm{s}\end{array}$ & $\begin{array}{r}\text { Cplex } \\
\text { /CBC }\end{array}$ & Cplex & CBC \\
\hline ST. ANALYSIS & & & & & & \\
Chorales-116 & 24 & $1: 24: 52$ & $0: 10: 18$ & 8.2 & $10,329,312$ & 20,382 \\
Balloons76 & 10 & $0: 00: 10$ & $0: 00: 14$ & 71.4 & 40,481 & 4 \\
BCW-367 & 8 & $0: 08: 33$ & $0: 00: 13$ & 39.4 & 79,980 & 463 \\
BCW-683 & 10 & $2: 02: 29$ & $0: 00: 32$ & 229.7 & 399,304 & 671 \\
WPBC-194 & 5 & $0: 57: 17$ & $0: 03: 32$ & 16.2 & 806,188 & 26,439 \\
Breast-Cancer-400 & 6 & $0: 02: 50$ & $0: 00: 16$ & 1062 & 181,990 & 1 \\
Glass-163 & 13 & $0: 56: 17$ & $0: 00: 05$ & 675.4 & $3,412,702$ & 64 \\
Horse-colic-151 & 5 & $0: 04: 50$ & $0: 00: 23$ & 12.6 & 135,018 & 2,184 \\
Iris-150 & 18 & $0: 09: 29$ & $0: 01: 10$ & 8.1 & 970,659 & 1,290 \\
Credit-300 & 8 & $0: 19: 35$ & $0: 00: 02$ & 587.5 & 176,956 & 66 \\
Lymphography-142 & 5 & $0: 00: 11$ & $0: 00: 01$ & 11.0 & 8,157 & 106 \\
Mech-analysis-107 & 7 & $0: 00: 05$ & $0: 00: 01$ & 5.0 & 11,101 & 68 \\
Mech-analysis-137 & 18 & $0: 07: 44$ & $0: 00: 27$ & 17.2 & 938,088 & 1,888 \\
Monks-tr-122 & 13 & $0: 02: 05$ & $0: 00: 05$ & 25.0 & 262,431 & 357 \\
Pb-gr-txt-198 & 11 & $0: 04: 21$ & $0: 00: 05$ & 52.2 & 135,980 & 110 \\
Pb-pict-txt-444 & 7 & $0: 02: 07$ & $0: 00: 02$ & 63.5 & 71,031 & 1,026 \\
Pb-hl-pict-277 & 10 & $0: 04: 17$ & $0: 00: 27$ & 9.5 & 22,047 & 115 \\
Postoperative-88 & 16 & $0: 15: 16$ & $0: 00: 01$ & 916.0 & $2,282,109$ & 171 \\
BV-OS-282 & 6 & $0: 05: 13$ & $0: 00: 24$ & 13.0 & 56,652 & 1,044 \\
Opel-Saab-80 & 6 & $0: 01: 03$ & $0: 00: 13$ & 4.8 & 87,542 & 7,314 \\
Bus-Van-437 & 6 & $0: 09: 17$ & $0: 00: 28$ & 19.9 & 55,224 & 6,795 \\
HouseVotes84-435 & 6 & $0: 04: 59$ & $0: 00: 11$ & 27.2 & 42,928 & 734 \\
Water-treat-206 & 4 & $0: 01: 10$ & $0: 00: 06$ & 11.7 & 12,860 & 482 \\
Water-treat-213 & 5 & $0: 17: 00$ & $0: 00: 51$ & 20.0 & 168,656 & 4,036 \\
\hline MAP LABELL. & & & & & & \\
CMS 600-1 & 600 & $1: 08: 41$ & $0: 04: 34$ & 15.0 & 110,138 & 14 \\
\hline TOTAL/MEAN & - & $\mathbf{8 : 2 9 : 5 1}$ & $\mathbf{0 : 2 4 : 1 1}$ & $\mathbf{2 1 . 1}$ & $\mathbf{2 0 , 7 9 7 , 5 3 4}$ & $\mathbf{6 4 , 3 7 3}$ \\
\hline
\end{tabular}

Table 1: Problems solved to proven optimality by both Cplex and CBC 


\begin{tabular}{|c|c|c|c|c|c|c|c|}
\hline \multirow{2}{*}{$\begin{array}{l}\text { Subset } 2 \\
\text { File Name }\end{array}$} & \multicolumn{5}{|c|}{ Cplex } & \multicolumn{2}{|c|}{ CBC } \\
\hline & $\begin{array}{c}\text { time } \\
h: m: s\end{array}$ & $\begin{array}{c}\text { best } \\
\text { sol. }\end{array}$ & $\begin{array}{r}\text { best } \\
\text { bound }\end{array}$ & $\begin{array}{l}\text { gap } \\
(\%)\end{array}$ & $\begin{array}{r}\text { mem } \\
\text { MB }\end{array}$ & opt & $\begin{array}{r}\text { time } \\
h: m: s\end{array}$ \\
\hline \multicolumn{8}{|l|}{ ST. ANALYSIS } \\
\hline \multirow[t]{2}{*}{ Chorales-134 } & $0: 36: 23$ & 33 & 16.0 & 51 & 371 & 30 & $0: 36: 23$ \\
\hline & $3: 00: 58$ & 30 & 21.1 & 30 & 992 & & \\
\hline \multirow[t]{2}{*}{ Chorales-107 } & $0: 04: 19$ & 28 & 12.1 & 57 & 61 & 27 & 0:04:19 \\
\hline & $3: 01: 27$ & 27 & 22.2 & 18 & 711 & & \\
\hline \multirow[t]{2}{*}{ Breast-Cancer-600 } & $0: 00: 13$ & 108 & 1.5 & 99 & 9 & 16 & $0: 00: 13$ \\
\hline & $3: 00: 11$ & 16 & 13.2 & 18 & 45 & & \\
\hline \multirow[t]{2}{*}{ Bridges-132 } & $0: 03: 39$ & 33 & 5.1 & 85 & 44 & 23 & $0: 03: 39$ \\
\hline & 3:01:09 & 23 & 10.0 & 56 & 1406 & & \\
\hline \multirow[t]{2}{*}{ Mech-analysis- 152} & $0: 34: 12$ & 22 & 12.1 & 45 & 328 & 21 & $0: 34: 12$ \\
\hline & $3: 00: 50$ & 21 & 16.1 & 24 & 865 & & \\
\hline \multirow[t]{2}{*}{ Monks-tr-124 } & $0: 01: 55$ & 27 & 8.1 & 70 & 25 & 24 & $0: 01: 55$ \\
\hline & $3: 00: 35$ & 24 & 20.0 & 17 & 381 & & \\
\hline \multirow[t]{2}{*}{ Monks-tr-115 } & $0: 04: 16$ & 29 & 9.1 & 69 & 67 & 27 & $0: 04: 16$ \\
\hline & $3: 01: 07$ & 27 & 19.0 & 30 & 1131 & & \\
\hline \multirow[t]{2}{*}{ Solar-flare-323 } & 0:00:04 & 51 & 5.0 & 90 & 18 & 38 & 0:00:04 \\
\hline & $3: 00: 45$ & 43 & 17.0 & 61 & 977 & & \\
\hline \multirow[t]{2}{*}{ BV-OS-376 } & 0:09:04 & 9 & 3.1 & 65 & 9 & 9 & 0:09:04 \\
\hline & $3: 00: 10$ & 9 & 6.0 & 33 & 56 & & \\
\hline \multirow[t]{2}{*}{ BusVan-445 } & $0: 10: 31$ & 13 & 3.0 & 77 & 11 & 8 & $0: 10: 31$ \\
\hline & $3: 00: 06$ & 9 & 5.1 & 43 & 56 & & \\
\hline TOTAL/MEAN & $30: 07: 18$ & gap & same & $=71^{\circ}$ & end & $33 \%$ & $1: 44: 36$ \\
\hline \multicolumn{8}{|l|}{ MAP LABELL. } \\
\hline \multirow[t]{2}{*}{ CMS 600-0 (4S) } & $0: 04: 27$ & 592 & 600 & 1.35 & 18 & 600 & $0: 04: 27$ \\
\hline & 3:03:00 & 594 & 600 & 1.01 & 770 & & \\
\hline \multirow[t]{2}{*}{ CMS 650-0 (4S) } & $0: 06: 26$ & 638 & 650 & 1.88 & 20 & 649 & $0: 06: 26$ \\
\hline & $3: 02: 34$ & 646 & 650 & 0.62 & 480 & & \\
\hline \multirow[t]{2}{*}{ CMS 650-1 (4S) } & $0: 04: 50$ & 647 & 650 & 0.46 & 7 & 649 & $0: 04: 50$ \\
\hline & $3: 03: 13$ & 648 & 650 & 0.31 & 904 & & \\
\hline \multirow[t]{2}{*}{ CMS 700-1 (4S) } & $0: 13: 06$ & 686 & 700 & 2.04 & 58 & 699 & $0: 13: 06$ \\
\hline & $3: 03: 00$ & 691 & 700 & 1.30 & 1045 & & \\
\hline \multirow[t]{2}{*}{ CMS 750-1 (4S) } & $0: 07: 53$ & 738 & 750 & 1.63 & 28 & 750 & $0: 07: 53$ \\
\hline & $3: 02: 19$ & 741 & 750 & 1.21 & 521 & & \\
\hline \multirow{2}{*}{ CMS 750-4 (4S) } & $0: 07: 05$ & 736 & 750 & 1.90 & 28 & 748 & $0: 07: 05$ \\
\hline & $3: 00: 24$ & 743 & 750 & 0.94 & 417 & & \\
\hline CMS 800-0 (4S) & $0: 19: 15$ & 773 & 800 & 3.49 & 55 & 798 & $0: 19: 15$ \\
\hline & $3: 02: 16$ & 773 & 800 & 3.49 & 533 & & \\
\hline CMS 800-1 (4S) & $0: 22: 24$ & 784 & 800 & 2.04 & 92 & 800 & $0: 22: 24$ \\
\hline & $3: 02: 30$ & 786 & 800 & 1.78 & 761 & & \\
\hline Railway & $0: 00: 31$ & 95 & 103 & 8.42 & 1 & 100 & $0: 00: 31$ \\
\hline & $3: 00: 02$ & 100 & 101 & 1.00 & 19 & & \\
\hline CMS 600-0 (4P) & 0:00:01 & 543 & 600 & 10.5 & 2 & 600 & 0:00:04 \\
\hline & $3: 02: 57$ & 574 & 600 & 4.53 & 782 & & \\
\hline CMS 600-1 (4P) & $0: 39: 07$ & 565 & 600 & 6.19 & 184 & 597 & $0: 39: 07$ \\
\hline & $3: 02: 55$ & 568 & 600 & 5.63 & 831 & & \\
\hline TOTALS & $33: 25: 10$ & gaps: & same $t$ & $=3.6 \%$ & end $=$ & $.0 \%$ & $2: 05: 4.1$ \\
\hline
\end{tabular}

Table 2: Problems solved to proven optimality by CBC but not by Cplex 


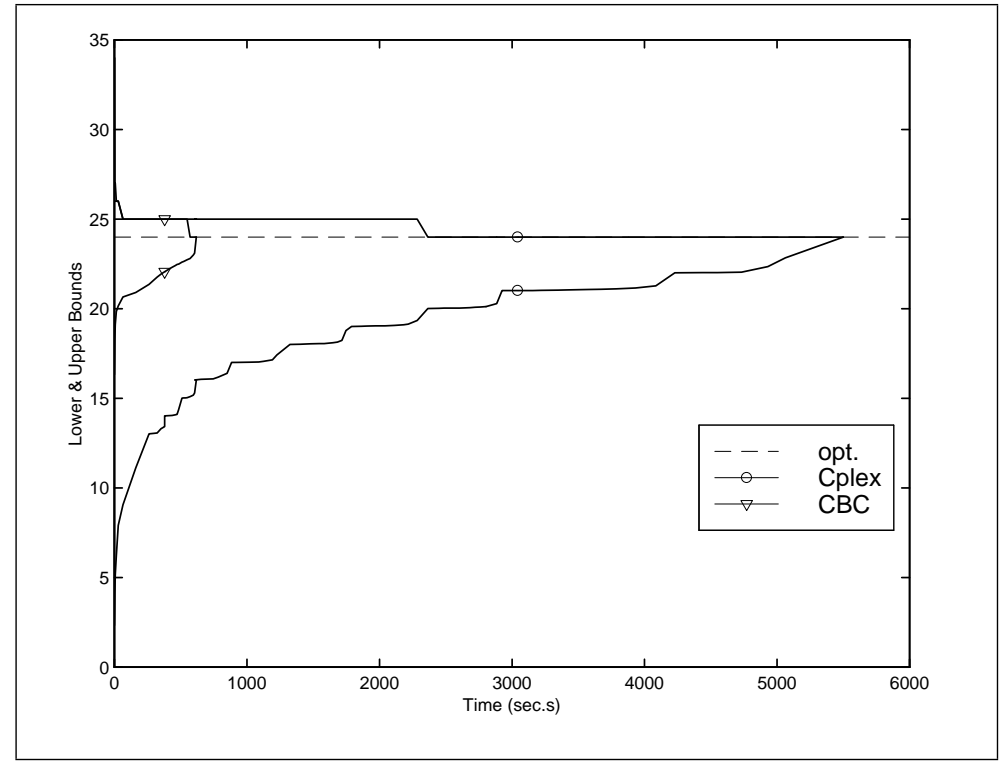

Figure 1: Optimizing the statistical-analysis instance Chorales-116 (minimization problem)

sample instances of this second subset.

For this class, the difference between CBC and Cplex is even more evident: not only Cplex was not able to solve any of these problems within the imposed time limit, but the best-bound trend suggests it is very unlikely it will converge even allowing for an extremely large computational effort. On the whole, CBC solved all the statistical analysis instances in less than 2 hours while, after the same time, Cplex had an average gap of more than $70 \%$. Moreover, we found that after 28 additional hours of computation the gap would have been only halved. An analogous behavior was observed on map labelling instances, for which the gap is not even halved after more than 31 additional hours of computation.

The results for the third subset are summarized in Table 3, where we report both for Cplex and $\mathrm{CBC}$ the same information given for Cplex in Table 2, excluding only the time column (as all instances reached the 3-hour time limit). In addition, in the last column of the we report the difference between the Cplex and CBC gaps $(\Delta G a p)$; in all cases, CBC obtained significantly smaller gaps.

\section{Conclusions}

We have proposed and analyzed computationally an automatic MIP reformulation method, aimed at removing the model dependency on the big-M 


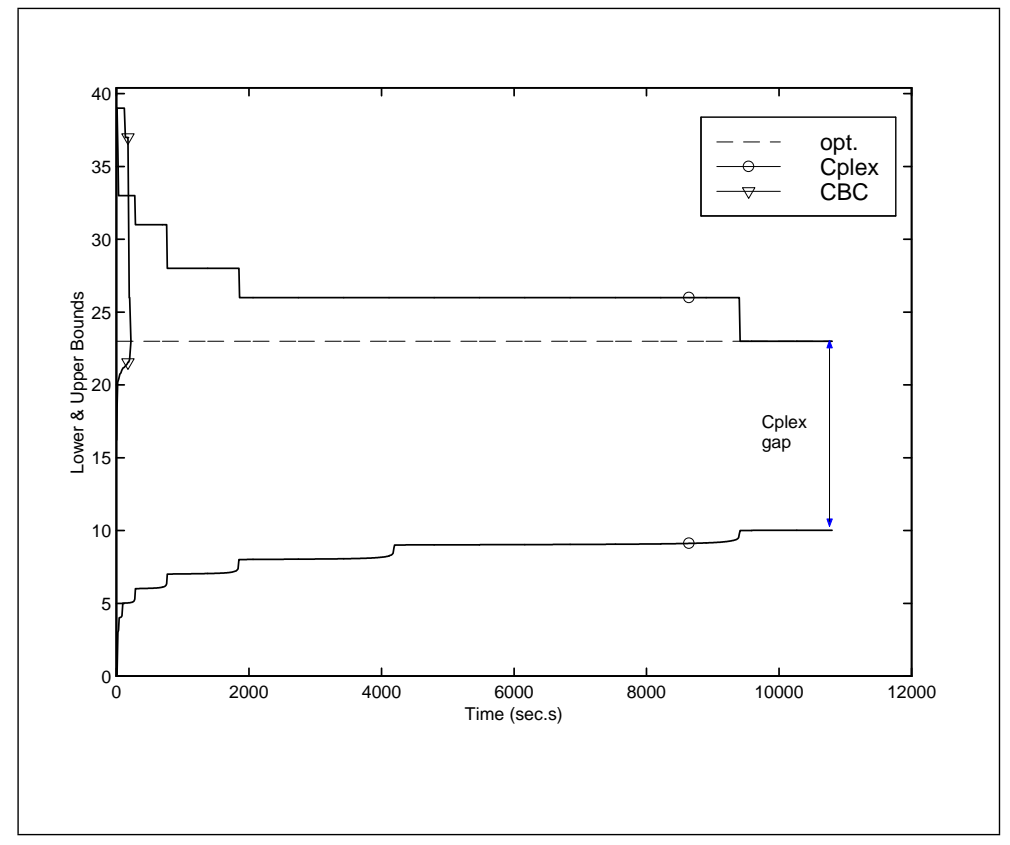

Figure 2: Optimizing the statistical-analysis instance Bridges-132 (minimization problem)

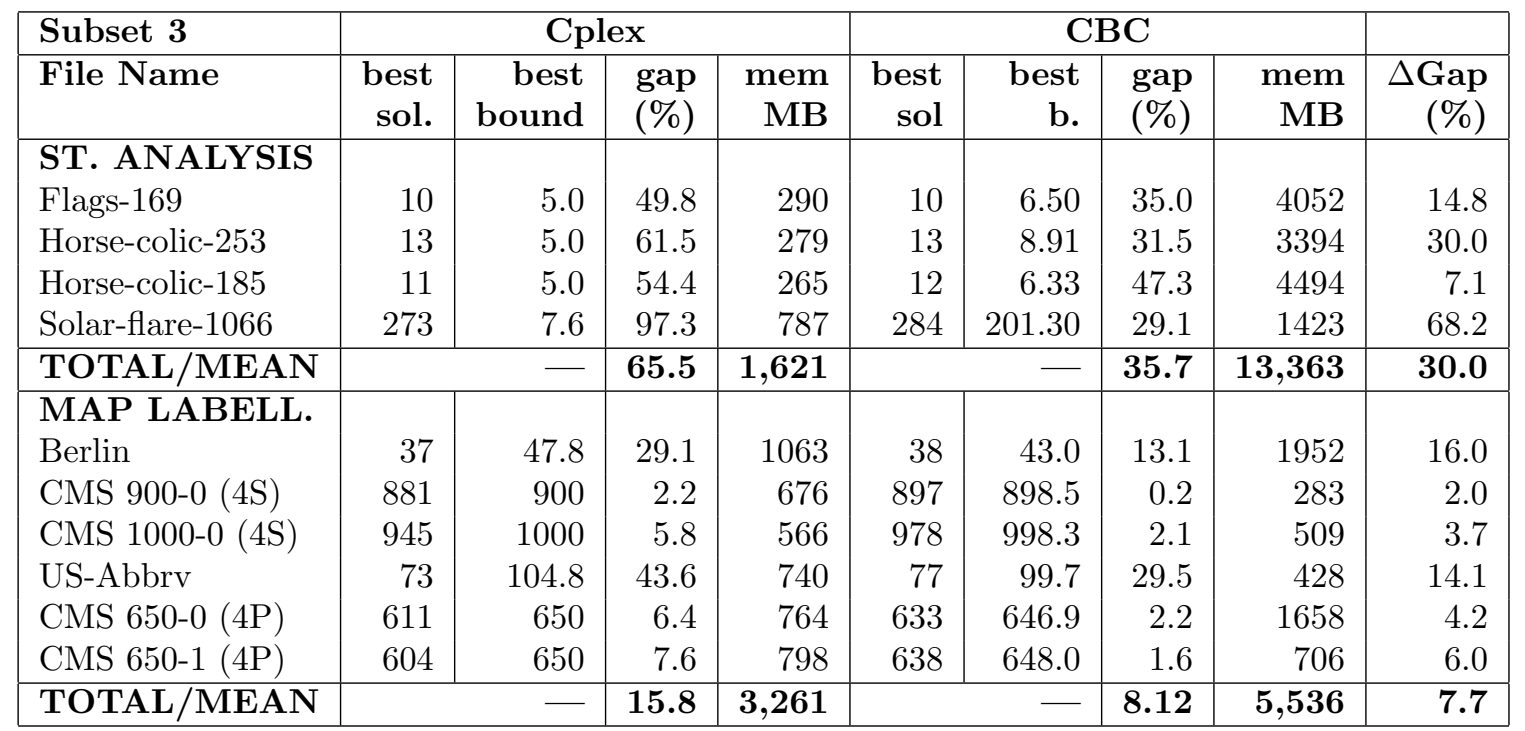

Table 3: Problems solved to proven optimality by neither codes 
coefficients often used to model logical implications.

Our technique is based on Hooker's idea of deriving Benders' cuts from minimal sets of inconsistencies, as proposed in [19]. In this respect, our main contributions have been (a) to present a separation heuristic that finds a minimal Benders' cuts for the special case of conditional constraints with linear implications, and (b) to test these cuts computationally on some hard MIP problems. This is an important special case because conditional constraints of this form are a very useful modeling device.

Our method proved particularly suited for the MIP's whose objective function only depends on the integer variables, and the continuous variables are linked to the integer ones through linear constraints involving a single binary variable each-typically multiplied by a large coefficient. This is precisely the situation arising in many important combinatorial problems modelled by the big-M technique, where the continuous variables are only used to certify a certain property of the feasible solutions (e.g., time-windows in scheduling problems, piece non-overlapping in nesting problems, etc.)

Computational results on two specific classes of hard-to-solve MIP's (namely, Statistical Analysis and Map Labelling problems) show that the new method produces automatically a reformulation which can be solved some orders of magnitude faster than the original MIP model.

Future direction of work should address the more general case where the MIP objective function depends on both the continuous and the integer variables, and analyze computationally the merits of the resulting technique. Some preliminary results in this directions are encouraging.

Finally, one could ask whether it is indeed convenient to use our approach as a decomposition method, rather than simply as a cut generation strategy. To this end, one could simply leave the continuous variables (and the associated constraints) in the master problem, and use the generated CB cuts as cutting planes. According to our computational experience, however, this approach has the main drawback of producing wild fractional points that are very difficult to separate by means of our simple CB-cut separation heuristics. Instead, preliminary computational tests seem to indicate that, for some problems, a better strategy would be to use our decomposition approach as a preprocessing tool. To be more specific, one could first run our decomposition approach for a while, so as to generate a large number of globally-valid CB cuts, and then restart the optimization of the original model with these cuts used to initialize the internal cut pool. The applicability of this 2-step approach is left to future investigation.

\section{Acknowledgements}

Work supported by MIUR and CNR, Italy. We thank two anonymous referees whose suggestions helped us in setting our contribution in the ap- 
propriate Constraint Programming context.

\section{References}

[1] E. Amaldi, M.E. Pfetsch, L.E. Trotter, "On the Maximum Feasible Subsystem Problem, IISs and IIS-Hypergraphs", Mathematical Programming 95, 3, 533-554, 2003.

[2] G. Andreello, A. Caprara, M. Fischetti, "Embedding Cuts within a Branch \& Cut Framework: a Computational Study with $\left\{0, \frac{1}{2}\right\}$-cuts", Technical Report, DEI, University of Padova, 2003.

[3] N. Ascheuer, M. Fischetti, M. Groetschel, "A Polyhedral Study of the Asymmetric Travelling Salesman Problem with Time Windows", Networks 36(2), 69-79, 2000.

[4] N. Ascheuer, M. Fischetti, M. Groetschel, "Solving the Asymmetric Travelling Salesman Problem with Time Windows by Branch-and-Cut", Mathematical Programming 90, 475-506, 2001

[5] J. F. Benders, "Partitioning Procedures for Solving Mixed Variables Programming Problems", Numerische Mathematik 4, 238-252, 1962.

[6] R. E. Bixby, M. Fenelon, Z. Gu, E. Rothberg, R. Wunderling, "MIP: Theory and Practice - Closing the Gap", available at www.ilog.com/products/optimization/tech/research/mip.pdf.

[7] C. Bliek, "Generalizing partial order and dynamic backtracking", Proceedings Fifteenth National Conference on Artificial Intelligence (AAAI-98). AAAI Press/MIT Press, 319-325, 1998.

[8] H. Cambazard, P.-E. Hladik, A.-M. Déplanche, N. Jussien, and Y. Trinquet, "Decomposition and learning for a hard real time task allocation algorithm", in M. Wallace, ed., Principles and Practice of Constraint Programming (CP 2004), Lecture Notes in Computer Science 3258, Springer, 2004.

[9] A. Caprara, M. Fischetti, " $\left\{0, \frac{1}{2}\right\}$ - Chvátal-Gomory Cuts", Mathematical Programming 74, 221-235, 1996.

[10] J. Chinneck, "Fast Heuristics for the Maximum Feasible Subsystem Problem", INFORMS J. Comput. 13 (3), 210-223, 2001.

[11] V. Chvátal, "Resolution Search", Discrete Applied Mathematis 73, 8199, 1997.

[12] G. Codato, "A Combinatorial Approach to Benders' Decomposition", Tesi di Laurea, University of Padova, 2003 (in italian). 
[13] N. De Siqueira, J. L., J.-F. Puget, "Explanation-based generalisation of failures", European Conference on Artificial Intelligence (ECAI-88), Pitman Publishers, Munich, 339-344, 1988.

[14] M.L. Ginsberg, "Dynamic backtracking", Journal of Artificial Intelligence Research 1, 25-46, 1993.

[15] M.L. Ginsberg, J. M. Crawford, D. W. Etherington, "Dynamic backtracking", CIRL, University of Oregon, 1996.

[16] M.L. Ginsberg, D. A. McAllester, "GSAT and dynamic backtracking", Second Workshop on Principles and Practice of Constraint Programming, 216-225, May 1994.

[17] J. Gleesonand, J. Ryan, "Identifying Minimally Infeasible Subsystems of Inequalities", ORSA Journal on Computing 2 (1), 61-63, 1990.

[18] F. Glover, L. Tangedhal, "Dynamic Strategies for Branch and Bound", Omega 4 (5), 571-576, 1976.

[19] J.N. Hooker, "Logic-based Methods for Optimization: Combining Opimization and Constraint Satisfaction", John Wiley \& Sons (2000).

[20] J.N. Hooker, "A hybrid method for planning and scheduling", in M. Wallace, ed., Principles and Practice of Constraint Programming (CP 2004), Lecture Notes in Computer Science 3258, Springer, 305-316, 2004.

[21] J.N. Hooker, M. A. Osorio, "Mixed logical/linear programming", Discrete Applied Mathematics 96-97, 395-442, 1999.

[22] J.N. Hooker, G. Ottosson, "Logic-based Benders Decomposition", Mathematical Programming, 96, 33-60, 2003.

[23] J.N. Hooker, Hong Yan, "Logic circuit verification by Benders decomposition," in V. Saraswat and P. Van Hentenryck, eds., Principles and Practice of Constraint Programming: The Newport Papers, MIT Press (Cambridge, MA) 267-288, 1995.

[24] ILOG Cplex 8.1: User's Manual and Reference Manual, ILOG, S.A., http://www.ilog.com/, 2003.

[25] ILOG Concert Technology 1.2: User's Manual and Reference Manual, ILOG, S.A., http://www.ilog.com/, 2003.

[26] V. Jain, I.E. Grossmann, "Algorithms for hybrid MILP/CP models for a class of optimization problems", INFORMS Journal on Computing $13,258-276,2001$. 
[27] U. Junker, Quickxplain, "Conflict detection for arbitrary constraint propagation algorithms," IJCAI01 Workshop on Modeling and Solving Problems with Constraints (CONS-1), Seattle, 2001.

[28] H-J. Kim, J.N. Hooker, "Solving Fixed-Charge Network Flow Problems with a Hybrid Optimization and Constraint Programming Approach", Annals of Operations Research, vol. 115 (1-4), 95-124, 2002.

[29] G. W. Klau, P. Mützel, "Optimal Labelling of Point Features in Rectangular Labelling Models", Mathematical Programming Ser. B, vol. 94 (2-3), 435-458, 2003.

[30] M. Parker, J. Ryan, "Finding the Minimum Weight IIS Cover of an Infeasible System of Linear Inequalities", Ann. Math. Artificial Intelligence 17, 107-126, 1996.

[31] P.A. Rubin, "Solving Mixed Integer Classification Problem by Decomposition", Annals of Operations Research 74, 51-64, 1997.

[32] E.S. Thorsteinsson, "Branch-and-Check: A Hybrid Framework Integrating Mixed Integer Programming and Constraint Logic Programming", Seventh International Conference on Principles and Practice of Constraint Programming (CP2001), 2001.

[33] P.M. Murphy, D. W. Aha, "UCI Repository of Machine Learning Databases", University of California, Department of Information and Computer Science, Irvine, CA, 1994; available at http://www.ics.uci.edu/ ${ }^{m l e a r n / M L R e p o s i t o r y . h t m l ~}$ 